\title{
Aromatic, or Antiaromatic, That Is the Question:
}

\author{
Ben Joseph R. Cuyacot ${ }^{1,2}$, Cina Foroutan-Nejad ${ }^{3 *}$
}

1. CEITEC - Central European Institute of Technology, Masaryk University, Kamenice 5/A4, CZ-62500 Brno, Czechia

2. Department of Chemistry, Faculty of Science, Masaryk University, Kamenice 5, CZ-62500 Brno, Czechia

3. Institute of Organic Chemistry, Polish Academy of Sciences, Kasprzaka 44/52, 01-224 Warsaw, Poland

Corresponding author's email: cina.foroutan-nejad@icho.edu.pl 
The term aromaticity in chemistry is reminiscent of symmetric, stable structures that have delocalized electrons and sustain diamagnetic ring currents in the presence of external magnetic fields. The two latter characteristics sometimes mistakenly are thought to be the same. ${ }^{1}$ In a groundbreaking discovery Liddle and co-workers synthesized a $\mathrm{D}_{3 h}$ complex with rare Th-Th bonds. ${ }^{2}$ Studying the electronic structure of a neutral singlet model system, $\mathbf{3}$ ", showed that the molecule has a delocalized HOMO between three Th atoms akin to a 2-electron 3-center bond. Employing nucleus independent chemical shift (NICS) ${ }^{3}$ computations and according to the pattern of the NICS scan $\operatorname{plot}^{4}$ on 3 ", the authors suggested that the molecule is a $\sigma$-aromatic species as it is reflected also in the title of their paper. Unfortunately, NICS is an inappropriate tool for evaluation of the magnetic aromaticity among early and mid-transition metals (TM), the elements that share a characteristic with thorium; they all have low-energy unoccupied orbitals. ${ }^{5-8}$ In the following we explain why NICS fails to assess aromaticity of molecules containing TMs and prove that the $\mathrm{Th}_{3}$ ring is a weakly antiaromatic system. We introduce a simple protocol to examine the aromaticity of any molecule utilizing software packages, which have been used by the authors of the main article.

NICS is a local measure of the strength of the magnetically induced currents. It is negative (shielding) around the inner rim of aromatic rings like benzene and positive (deshielding) around their periphery because of the diamagnetic ring currents. The shielding/deshielding regions reverse in the case of antiaromatic rings because of the paramagnetic ring currents. ${ }^{3}$ Many TMs, lanthanides, and actinide atoms sustain strong local paramagnetic currents akin to the antiaromatic rings; therefore, their neighboring atoms (or ghost atoms that are probes of NICS computation) are strongly influenced depending on where they lie on the shielding/deshielding regions of the paramagnetic local currents. In a cyclic structure containing several TMs the center of the ring experiences a strong shielding because of the local paramagnetic current around the metal atoms. 
This shielding effect disappears a few angstroms above/below the ring plane and replaces with deshielding arising from the local paramagnetic current cones around the atoms, Figure 1a.

Atomic and bond magnetizabilities ${ }^{9}$ are two probes in the toolbox of the quantum theory of atoms in molecules (QTAIM) ${ }^{10}$ that provide a quick test for assessing the effects of local currents around the atoms and the ring current, respectively. ${ }^{11}$ A negative value designates the presence of diamagnetic current and vice versa. The out-of-plane component of the atomic and bond magnetizability like the out-of-plane NICS, $\mathrm{NICS}_{\mathrm{zz}}$, is a more sensitive probe to assess the nature of the electronic currents. ${ }^{11}$ The out-of-plane atomic and bond magnetizabilities of Th, and Th-Th are -1.2 and +9.8 cgs-ppm. In comparison, the out-of-plane bond magnetizabilities of benzene and cyclohexane are -5.0 and -1.5 cgs-ppm, respectively, at the same level of theory. The atomic magnetizabilities suggest the Th atoms in 3" sustain a strong local paramagnetic current. However, the dimagnetic ring current, associated with aromaticity is absent according to the bond magnetizability.

Visualization of the isosurfaces and profiles of the current density is another useful tool to assess the presence/absence of the ring current, irrespective of their diamagnetic or paramagnetic nature. If the isosurface values are selected properly, they can even roughly show the responsible atomic/molecular orbitals involving in the ring current. ${ }^{8}$ An isosurface value of 0.0005 au is a safe choice to reveal an strong aromatic/antiaromatic ring currents. ${ }^{8}$ In 3 " this isosurface value (even 0.0003 au, Figure S1) does not verify the presence of any global current around the $\mathrm{Th}_{3}$ moiety, Figure 1 b and c (see Figure S2 for comparison with benzene). Similarly, a profile of the current density on the ring plane of $\mathrm{Th}_{3}$ shows the dominance of the paramagnetic currents, Figure $1 \mathbf{d}$, unlike benzene, Figure S2. 


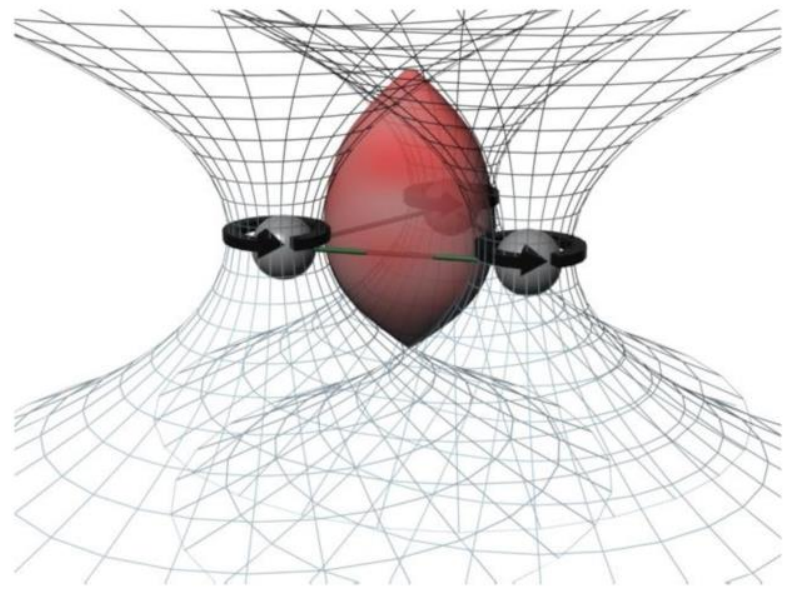

a

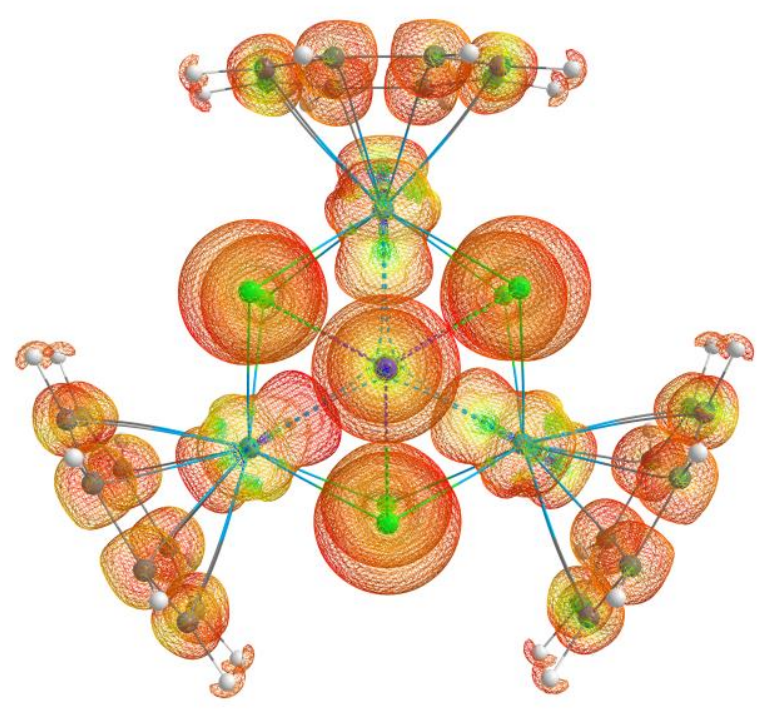

C

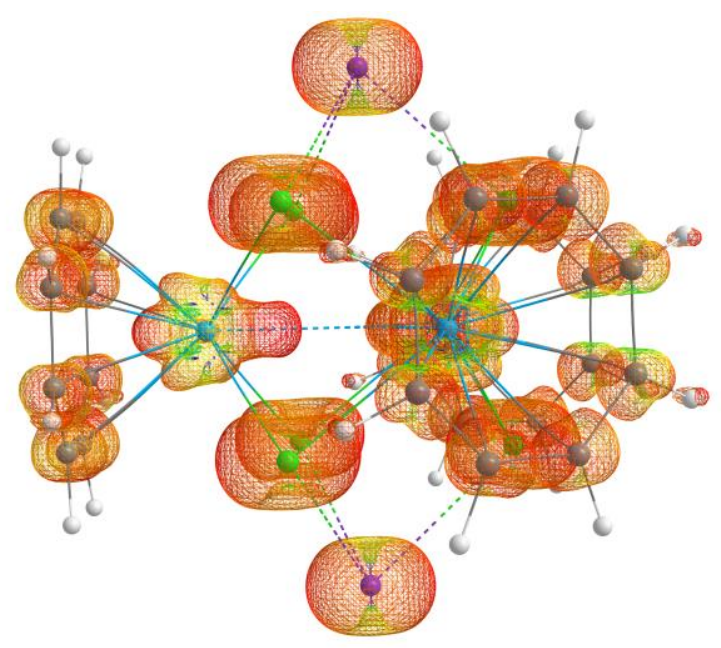

b

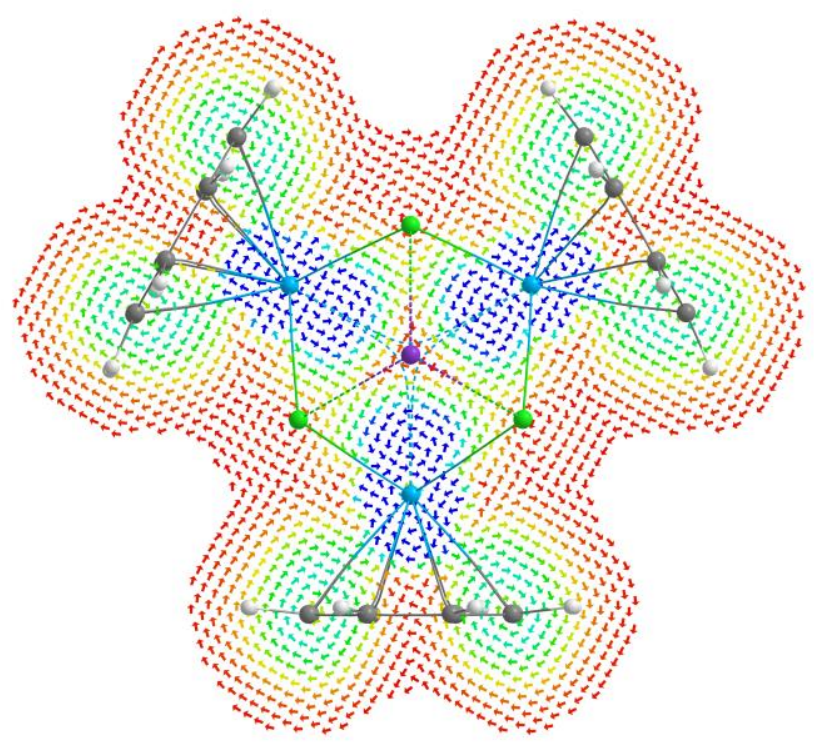

d

Figure 1. (a) local paramagnetic currents (black arrows) induce a conical region of deshielding in the presence of a magnetic field applied perpendicular to the ring structure. Outside of this cone, the net effect of the paramagnetic current is shielding. The red region in the center depicts the induced shielding as a result of the local paramagnetic currents (this entry is adopted with permission from reference 6). The isosurfaces of current density $(\mathrm{J}(\mathrm{r})=0.0005 \mathrm{au})$ for a magnetic field applied perpendicular to the ring plane from the side (b) and top (c) views do not show any connection between the neighboring Th atoms. This proves the lack of any strong dia- or paramagnetic ring current. (d) The profile of the current density on the ring plane of $\mathrm{Th}_{3}$. The blue to red color shows strong $(\mathrm{J}(\mathrm{r}) \geq 0.0005 \mathrm{au})$ to weak $(\mathrm{J}(\mathrm{r})=0.0000)$. The strong currents appear around the Th atoms. A paramagnetic (counterclockwise) current around the inner rim of the $\mathrm{Th}_{3}$ framework is verifiable. Only the weakest currents (in red) appear diamagnetic (clockwise). 
Up to this point, all tasks can be done using AIMAll suite of programs, ${ }^{12}$ which has been used by Liddle and co-workers to analyze bonding in 3". The most reliable measure of the magnetic aromaticity is the integrated current intensity, passing through a plane perpendicular to the ring plane and cutting through a bond. Often one can integrate current intensity passing through an interatomic surface (IAS) of QTAIM but sometimes this is not possible due to the absence of an IAS, or complex molecular topology. ${ }^{7}$ In the case of $\mathbf{3}$ " the complex topology of the molecule makes a reliable computation of the current density passing through the IAS impossible; see Methods for details and a simple approach to bypass this issue. Therefore, we performed this task using ReSpect ${ }^{13,14}$ software at the scalar and fully relativistic levels, Table $\mathbf{1 .}$

Table 1. The ring current and the contribution of the HOMO in the ring current in $\mathrm{nA} . \mathrm{T}^{-1}$ units in 3". Negative current values correspond to the paramagnetic current that is associated with magnetic antiaromaticity. (1c) and (4c) refer to scalar and fully relativistic levels of theory.

\begin{tabular}{lllll} 
& PBE (1c) & PBE (4c) & PBE0 (1c) & PBE0 (4c) \\
\hline Ring Current & -1.23 & -1.20 & -1.66 & -0.93 \\
HOMO & -3.54 & -3.98 & -2.36 & -2.90 \\
\hline
\end{tabular}

The contribution of the individual molecular orbital in the current density of $\mathbf{3}$ " was measured by placing a rectangular plane $\pm 10 \AA$ above and below the $\mathrm{Th}_{3}$ ring and from the center to $20 \AA$ away from the center cutting through a Th-Th bond at scalar relativistic (1c) and fully relativistic (4c) levels, Table 1. Alternatively, another rectangle with the same length but $2 \AA$ width ( $\pm 1.0 \AA$ above and below the ring plane) was used to measure the total $\sigma$-current density for all MOs passing in between $\mathrm{Th}-\mathrm{Th}$ bond. This rectangle was selected narrower than the previous one to make sure that the local current density of the potassium ions does not interfere with the ring current measurements. The total current density and the HOMO's current density correspond to 
those of a weakly antiaromatic molecule. The difference between scalar and fully relativistic currents is about $-0.44 \mathrm{nA} . \mathrm{T}^{-1}\left(12.5 \%\right.$ of the total scalar current) at PBE level and $0.54 \mathrm{nA} . \mathrm{T}^{-1}$ $(23 \%)$ at PBE0 level. A fully relativistic wave function suggests an intensified antiaromatic character.

Our analyses prove that 3 " is not aromatic and again proves that NICS cannot assess the nature of the ring current in metallic clusters. Unlike hydrocarbons such as cyclobutadiene, magnetic antiaromaticity among large rings and metallic clusters is not uncommon and does not necessarily imply energetic instability. ${ }^{6,15}$ Furthermore, as it has been discussed before, magnetic properties such as ring current and NICS are response properties that have no direct relationship with the ground-state properties such as total electronic energy of a molecule. ${ }^{1}$ Electron counting too is proved to be irrelevant to the energetic stability. ${ }^{16}$ The recently synthesized Th complex is a unique stable system with Th-Th bonds but this system sustains paramagnetic currents, which means it is magnetically antiaromatic.

\section{Acknowledgments}

Computational resources were provided by CESNET (LM2015042), the CERIT Scientific Clouds (LM2015085). This research was funded in whole by National Science Centre, Poland 2020/39/B/ST4/02022. For the purpose of Open Access, the author has applied a CC-BY public copyright license to any Author Accepted Manuscript (AAM) version arising from this submission.

\section{Data availability}

The isosurfaces of current density at $\mathrm{J}(\mathrm{r})=0.0003 \mathrm{au}$ (Fig. S1), the profile and isosurface plots of the current density of benzene at different values (Fig. S2), and details of computational methods employed to perform the present work are presented in Supporting Information. 


\section{Author contribution}

BJRC performed all computations with ReSpect including integration of current densities and assessing the MO contributions to the current intensity. CFN performed QTAIM analyses and wrote the paper.

\section{Competing interests}

The authors declare no competing interest

\section{References}

1. Badri, Z. \& Foroutan-Nejad, C. Unification of ground-state aromaticity criteria - structure, electron delocalization, and energy - in light of the quantum chemical topology. Phys. Chem. Chem. Phys. 18, 11693-11699 (2016).

2. Boronski, J. T. et al. A Crystalline Tri-thorium Cluster with $\sigma$-Aromatic Metal-Metal Bonding. Nature 1-7 (2021) doi:10.1038/s41586-021-03888-3.

3. Chen, Z., Wannere, C. S., Corminboeuf, C., Puchta, R. \& Schleyer, P. von R. NucleusIndependent Chemical Shifts (NICS) as an Aromaticity Criterion. Chem. Rev. 105, 3842-3888 (2005).

4. Stanger, A. Nucleus-Independent Chemical Shifts (NICS): Distance Dependence and Revised Criteria for Aromaticity and Antiaromaticity. J. Org. Chem. 71, 883-893 (2006).

5. Ramírez-Tagle, R., Alvarado-Soto, L., Arratia-Perez, R., Bast, R. \& Alvarez-Thon, L. Probing the aromaticity of the $[(\mathrm{HtAc}) 3(\mu 2-\mathrm{H}) 6],[(\mathrm{HtTh}) 3(\mu 2-\mathrm{H}) 6],+$, and $[(\mathrm{HtPa}) 3(\mu 2-\mathrm{H}) 6]$ clusters. J. Chem. Phys. 135, 104506 (2011).

6. Badri, Z. et al. All-Metal Aromaticity: Revisiting the Ring Current Model among Transition Metal Clusters. J. Chem. Theory Comput. 9, 4789-4796 (2013). 
7. Foroutan-Nejad, C. Is NICS a reliable aromaticity index for transition metal clusters? Theor Chem Acc 134, 8 (2015).

8. Foroutan-Nejad, C., Vícha, J. \& Ghosh, A. Relativity or aromaticity? A first-principles perspective of chemical shifts in osmabenzene and osmapentalene derivatives. Phys. Chem. Chem. Phys. 22, 10863-10869 (2020).

9. Keith, T. A. Atomic Response Properties. in The Quantum Theory of Atoms in Molecules 6194 (John Wiley \& Sons, Ltd, 2007). doi:10.1002/9783527610709.ch3.

10. Bader, R. F. W. Atoms in molecules: a quantum theory. (Clarendon Press, 1990).

11. Foroutan-Nejad, C. Interatomic Magnetizability: A QTAIM-Based Approach toward Deciphering Magnetic Aromaticity. J. Phys. Chem. A 115, 12555-12560 (2011).

12. Keith, T. A. AIMAll. (Gristmill Software, 2017).

13. Repisky, M. et al. Relativistic Spectroscopy DFT Program ReSpect, Developer Version 5.0.0. (2018).

14. Repisky, M. et al. ReSpect: Relativistic spectroscopy DFT program package. J. Chem. Phys. 152, 184101 (2020).

15. Conradie, J., Foroutan-Nejad, C. \& Ghosh, A. Norcorrole as a Delocalized, Antiaromatic System. Scientific Reports 9, 4852 (2019).

16. Zhao, L., Grande-Aztatzi, R., Foroutan-Nejad, C., Ugalde, J. M. \& Frenking, G. Aromaticity, the Hückel 4 n+2 Rule and Magnetic Current. ChemistrySelect 2, 863-870 (2017). 